\title{
Approximate controllability of semilinear strongly damped wave equation with impulses, delays, and nonlocal condi- tions
}

\author{
Cosme Duque $^{\mathrm{a}}$, Jahnett Uzcátegui ${ }^{\mathrm{a}}$, Hugo Leiva ${ }^{\mathrm{b}, *}$, Oscar Camacho $^{\mathrm{c}}$ \\ a Departamento de Matemáticas, Universidad de Los Andes, Mérida 5101, Venezuela. \\ ${ }^{b}$ School of Mathematical and Computational Sciences, University YachayTech, San Miguel de Urcuqui, Imbabura, Ecuador. \\ ${ }^{c}$ Departamento de Automatización y Control Industrial, Escuela Politecnica Nacional, Quito, Ecuador.
}

\begin{abstract}
In this paper, we prove that the interior approximate controllability of the linear strongly damped wave equation is not destroyed if we add impulses, nonlocal conditions, and a nonlinear perturbation with delay in the state. Specifically, we prove the interior approximate controllability of the semilinear strongly damped wave equation with impulses, delays, and nonlocal conditions. This is done by applying Roth's Fixed Point Theorem and the compactness of the semigroup generated by the linear uncontrolled system. Finally, we present some open problems and a possible general framework to study the controllability of impulsive semilinear second-order diffusion process in Hilbert spaces with delays and nonlocal conditions.
\end{abstract}

Keywords: Interior approximate controllability, impulsive semilinear strongly damped wave equation with delays and nonlocal conditions, strongly continuous semigroups, Rothe's fixed point theorem.

2010 MSC: 93B05, 93C10.

(C)2020 All rights reserved.

\section{Introduction}

In this paper, we study the interior approximate controllability of the following strongly damped semilinear wave equations with delays

$$
\begin{aligned}
& w^{\prime \prime}+\eta(-\Delta)^{1 / 2} w^{\prime}+\gamma(-\Delta) w=1_{\omega} u(t, x)+f\left(t, w, w^{\prime}, w\left(t-r_{1}\right), \ldots, w\left(t-r_{m}\right),\right. \\
& \left.w^{\prime}\left(t-r_{1}\right) \ldots, w^{\prime}\left(t-r_{m}\right), u(t, x)\right) \text { in } \Omega_{\tau}
\end{aligned}
$$

in the space $Z^{1 / 2}=D\left((-\Delta)^{1 / 2}\right) \times L^{2}(\Omega)$ where $w^{\prime}=\frac{\partial w}{\partial t}, w^{\prime \prime}=\frac{\partial^{2} w}{\partial t^{2}}, \Omega \subset \mathbb{R}^{N}, N \geqslant 1$ is a bounded domain, $\gamma$ and $\eta$ are positive numbers. Along with Dirichlet boundary condition, nonlocal conditions,

\footnotetext{
*Corresponding author

Email addresses: duquec@ula.ve (Cosme Duque), jahnettu@ula.ve (Jahnett Uzcátegui), hleiva@yachaytech.edu.ec (Hugo

Leiva), oscar . camacho@epn. edu.ec (Oscar Camacho)
}

doi: $10.22436 /$ jmcs.020.02.04

Received: 2019-06-17 Revised: 2019-07-22 Accepted: 2019-08-07 
and impulses

$$
\begin{cases}w(t, x)=0, & \text { on } \Omega_{\partial}, \\ w(s, x)+h_{1}\left(w\left(\tau_{1}+s, x\right), \ldots, w\left(\tau_{q}+s, x\right)\right)=\phi_{1}(s, x), & \\ w^{\prime}(s, x)+h_{2}\left(w^{\prime}\left(\tau_{1}+s, x\right), \ldots, w^{\prime}\left(\tau_{q}+s, x\right)\right)=\phi_{2}(s, x), & \text { in } \Omega_{-r}, \\ w^{\prime}\left(t_{k}^{+}, x\right)=w^{\prime}\left(t_{k}^{-}, x\right)+I_{k}\left(t_{k}, w\left(t_{k}, x\right), w^{\prime}\left(t_{k}, x\right), u\left(t_{k}, x\right)\right), & k=1, \ldots, p .\end{cases}
$$

Here $\Delta$ denotes de Laplacian operator, $\Omega$ is a bounded domain in $\mathbb{R}^{N}(N \geqslant 1), \partial \Omega$ denotes the boundary of $\Omega, \Omega_{\tau}=(0, \tau] \times \Omega_{2}, \Omega_{\partial}=(0, \tau) \times \partial \Omega, \Omega_{-r}=[-r, 0] \times \Omega, \omega$ is an open nonempty subset of $\Omega$, $1_{\omega}$ denotes the characteristic function of the set $\omega$, the distributed control u belongs to $L_{2}\left([0, \tau] ; L_{2}(\Omega)\right)$, $\phi_{i}:[-r, 0] \times \Omega \longrightarrow \mathbb{R}, i=1,2$, are continuous functions, $0<r_{1}<\cdots<r_{m}<r$ are the delays, and $0<\tau_{1}<\cdots<\tau_{\mathrm{q}}<\tau$.

From now on, we shall assume the following hypotheses.

H1) The functions $I_{k}:[0, \tau] \times \mathbb{R} \times \mathbb{R} \times \mathbb{R} \longrightarrow \mathbb{R}, k=1, \ldots, p, f:[0, \tau] \times \mathbb{R} \times \mathbb{R} \times \mathbb{R}^{m} \times \mathbb{R}^{m} \times \mathbb{R} \longrightarrow \mathbb{R}$, and $h_{i}: \mathbb{R}^{q} \longrightarrow \mathbb{R}, i=1,2$ are smooth enough, such that the above problem admits solutions according with [15].

H2) The following estimates hold:

$$
\begin{aligned}
& \left|f\left(t, \xi, \vartheta, \xi_{1}, \ldots, \xi_{m}, \vartheta_{1}, \ldots \vartheta_{m}, u\right)\right| \leqslant a_{0}\left(|\xi|^{\alpha_{0}}+|\vartheta|^{\alpha_{0}}\right)+\sum_{l=1}^{m} a_{l}\left(\left|\xi_{l}\right|^{\rho_{l}}+\left|\vartheta_{l}\right|^{\rho_{l}}\right) \\
& +\mathrm{b}_{0}|u|^{\beta_{0}}+\mathrm{c}_{0}, \quad \mathrm{u}, \xi, \vartheta, \xi_{l}, \vartheta_{l} \in \mathbb{R}, \\
& \left|I_{k}(t, \xi, \vartheta, u)\right| \leqslant a_{k}\left(|\xi|^{\alpha_{k}}+|\vartheta|^{\alpha_{k}}\right)+b_{k}|u|^{\beta_{k}}+c_{k}, k=1, \ldots, p, u, \xi, \vartheta \in \mathbb{R}, \\
& \left|h_{i}\left(\xi_{1}, \ldots, \xi_{q}\right)\right| \leqslant \sum_{j=1}^{q} e_{k}^{i}\left|\xi_{j}^{i}\right|^{i} \zeta_{j}^{i}+e_{0}^{i}, \quad i=1,2, \quad\left(\xi_{1}, \ldots, \xi_{q}\right) \in \mathbb{R}^{q}, \\
& \left|h_{i}\left(\xi_{1}, \ldots, \xi_{q}\right)-h_{i}\left(\vartheta_{1}, \ldots \vartheta_{q}\right)\right| \leqslant K \max _{1 \leqslant j \leqslant q}\left|\xi_{j}-\vartheta_{j}\right|, \quad i=1,2, \quad \xi_{j}, \vartheta_{j} \in \mathbb{R}, \\
& \frac{1}{2} \leqslant \alpha_{k}, \beta_{k}<1, \quad k=0,1, \ldots, p, \\
& \frac{1}{2} \leqslant \rho_{l}<1, \quad l=1, \ldots, m \text {, } \\
& \frac{1}{2} \leqslant \zeta_{j}<1, \quad j=1, \ldots, q \text {, }
\end{aligned}
$$

and

$$
w\left(t_{k}, x\right)=w\left(t_{k}^{+}, x\right)=\lim _{t \rightarrow t_{k}^{+}} w(t, x), \quad w\left(t_{k}^{-}, x\right)=\lim _{t \rightarrow t_{k}^{-}} w(t, x) .
$$

To set this problem, we shall choose the following natural Banach space:

$$
\mathrm{PC}_{\mathrm{t}_{1} . . \mathrm{t}_{\mathrm{P}}}\left([-\mathrm{r}, \tau] ; \mathrm{Z}^{1 / 2}\right)=\left\{z: J=[0, \tau] \rightarrow \mathrm{Z}^{1 / 2}: z \in \mathrm{C}\left(\mathrm{J}^{\prime} ; \mathrm{Z}^{1 / 2}\right), \exists z\left(\mathrm{t}_{\mathrm{k}}^{+}, \cdot\right), z\left(\mathrm{t}_{\mathrm{k}}^{-}, \cdot\right) \text { and } z\left(\mathrm{t}_{\mathrm{k}}, \cdot\right)=z\left(\mathrm{t}_{\mathrm{k}}^{+}, \cdot\right)\right\},
$$

$J^{\prime}=[-r, \tau] \backslash\left\{t_{1}, t_{2}, \ldots, t_{p}\right\}$ endowed with the norm $\|z\|_{0}=\sup _{t \in[-r, \tau]}\|z(t, \cdot)\|_{Z^{1 / 2}}$, where $z=(w, v)^{\top}=$ $\left(w, w_{t}\right)^{\top}$ and

$$
\|z\|_{Z^{1 / 2}}=\left(\int_{\Omega}\left(\left\|(-\Delta)^{1 / 2} w\right\|^{2}+\|v\|^{2}\right) d x\right)^{1 / 2}, \text { for all } z \in Z^{1 / 2}
$$

Remark 1.1. It is clear that $P C_{t_{1} . . t_{P}}\left([-r, \tau] ; Z^{1 / 2}\right)$ is a closed subspace of the Banach space of all piecewise continuous functions $\mathrm{PC}\left([-r, \tau] ; Z^{1 / 2}\right)$ with the supreme norm, which implies that $\mathrm{PC}_{t_{1} . . t_{\mathrm{P}}}\left([-r, \tau] ; Z^{1 / 2}\right)$ is a Banach space with the same norm.

We note that the interior controllability of the following strongly damped wave equation without 
impulses, delays, and nonlocal conditions

$$
\begin{cases}w^{\prime \prime}+\eta(-\Delta)^{1 / 2} w^{\prime}+\gamma(-\Delta) w=1_{w} \mathrm{u}(\mathrm{t}, \mathrm{x}), & \text { in }(0, \tau] \times \Omega, \\ w=0, & \text { on }(0, \tau) \times \partial \Omega, \\ w(0, x)=w_{0}(x) \quad w^{\prime}(0, x)=w_{1}(x), & \text { in } \Omega,\end{cases}
$$

has been proved in [13], where some ideas are taken to study this present problem. Finally, the approximate controllability of the system (1.1) follows from the approximate controllability of (1.6), the compactness of the semigroup generated by the uncontrolled linear equations, the conditions (1.2)-(1.5) satisfied by $f, h_{1}, h_{2}, I_{k}$, and applying the following theorem.

Theorem 1.2 (Rothe's Fixed Theorem, $[1,10,26])$. Let $\mathrm{E}$ be a Banach space. Let $\mathrm{B} \subset \mathrm{E}$ be a closed convex subset such that the zero of $\mathrm{E}$ is contained in the interior of $\mathrm{B}$. Let $\Phi: \mathrm{B} \rightarrow \mathrm{E}$ be a continuous mapping with $\Phi(\mathrm{B})$ relatively compact in $\mathrm{E}$ and $\Phi(\partial \mathrm{B}) \subset \mathrm{B}$. Then there is a point $\mathrm{x}^{*} \in \mathrm{B}$ such that $\Phi\left(\mathrm{x}^{*}\right)=\mathrm{x}^{*}$.

Recently, there are many papers on evolution equations with impulses and delay or with impulses and nonlocal conditions or with local conditions and delays, where not only the controllability is studied, but also other aspects are studied, such as the existence of mild solutions, synchronization, stability, etc.. To mention, we have the following references: $[3-5,8,9,12,18,20,21,24,25]$.

\section{Abstract formulation of the problem.}

In this section, we choose a Hilbert space where system (1.1) can be written as an abstract differential equation; to this end, we shall use the following notations.

Let $X=U=\mathrm{L}^{2}(\Omega)=\mathrm{L}^{2}(\Omega, \mathbb{R})$ and consider the linear unbounded operator $A: D(A) \subset X \rightarrow X$ defined by

$$
A \varphi=-\Delta \varphi, \text { where } D(A)=H^{2}(\Omega, \mathbb{R}) \cap H_{0}^{1}(\Omega, \mathbb{R}) .
$$

The fractional powered spaces $X^{\alpha}$ (see details in [13]) are given by

$$
X^{\alpha}=D\left(A^{\alpha}\right)=\left\{x \in X: \sum_{n=1}^{\infty} \lambda_{n}^{2 \alpha}\left\|E_{n} x\right\|^{2}<\infty\right\},
$$

endowed with the norm

$$
\|x\|_{\alpha}=\left\|A^{\alpha} x\right\|=\left(\sum_{n=1}^{\infty} \lambda_{n}^{2 \alpha}\left\|E_{n} x\right\|^{2}\right)^{1 / 2},
$$

where $\left\{E_{j}\right\}$ is a family of complete orthogonal projections in $X$; and for the Hilbert sapce $Z^{\alpha}=X^{\alpha} \times X$ the corresponding norm is

$$
\left\|\left(\begin{array}{c}
w \\
v
\end{array}\right)\right\|_{Z^{\alpha}}=\sqrt{\|w\|_{\alpha}^{2}+\|v\|^{2}}
$$

Proposition 2.1. Given $j \geqslant 1$, the operator $P_{j}: Z^{\alpha} \longrightarrow Z^{\alpha}$ defined by

$$
P_{j}=\left[\begin{array}{cc}
E_{j} & 0 \\
0 & E_{j}
\end{array}\right]
$$

is a continuous (bounded) orthogonal projection in the Hilbert space $Z^{\alpha}$.

Hence, the equation (1.1) can be written as an abstract second order ordinary differential equation in $\mathrm{X}$ as

$$
\left\{\begin{array}{cl}
w^{\prime \prime}+\eta A^{1 / 2} w^{\prime}+\gamma A w=B_{\omega} u+f^{e}\left(t, w(t), w^{\prime}(t), w_{t}\left(-r_{1}\right), \ldots, w_{t}\left(-r_{m}\right),\right. & \\
\left.w_{t}^{\prime}\left(-r_{1}\right), \ldots, w_{t}^{\prime}\left(-r_{m}\right), u(t)\right), & t \in(0, \tau], t \neq t_{k}, \\
w(s)+g_{1}\left(w_{\tau_{1}}, \ldots, w_{\tau_{q}}\right)(s)=\phi_{1}(s), \quad w(s)+g_{2}\left(w_{\tau_{1}}, \ldots, w_{\tau_{q}}\right)(s)=\phi_{2}(s), & s \in[-r, 0] \\
w^{\prime}\left(t_{k}^{+}\right)=w^{\prime}\left(t_{k}\right)+I_{k}^{e}\left(t_{k}, w\left(t_{k}\right), w^{\prime}\left(t_{k}\right), u\left(t_{k}\right)\right), & k=1, \ldots, p
\end{array}\right.
$$


where

$$
\begin{aligned}
& \mathrm{I}_{\mathrm{k}}^{\mathrm{e}}:[0, \tau] \times \mathrm{Z}^{1 / 2} \times \mathrm{U} \longrightarrow \mathrm{X}, \\
& (\mathrm{t}, w, v, \mathrm{u})(\cdot) \longmapsto \mathrm{I}_{\mathrm{k}}(\mathrm{t}, w(\cdot), v(\cdot), \mathrm{u}(\cdot)), \\
& f^{e}:[0, \tau] \times Z^{1 / 2} \times C^{m}\left([-r, 0] ; Z^{1 / 2}\right) \times C^{m}\left([-r, 0] ; Z^{1 / 2}\right) \times U \longrightarrow X \\
& \left(t, w, v, \varphi_{1}, \ldots, \varphi_{m}, \psi_{1}, \ldots, \psi_{m}, u\right)(\cdot) \longmapsto \begin{array}{c}
f\left(t, w(\cdot), v(\cdot), \varphi_{1}\left(-r_{1}, \cdot\right), \ldots \varphi\left(-r_{m}, \cdot,\right),\right. \\
\left.\psi_{1}\left(-r_{1}, \cdot\right), \ldots, \psi_{m}\left(-r_{m}, \cdot\right), u(\cdot)\right),
\end{array} \\
& \mathrm{B}_{\omega}: \mathrm{U} \longrightarrow \mathrm{U} \text {, } \\
& \mathfrak{u}(\cdot) \longmapsto 1_{\omega} u(\cdot), \\
& g_{i}: C^{q}([-r, 0] ; X) \longrightarrow C([-r, 0] ; X), \\
& g_{i}\left(\varphi_{1}, \ldots, \varphi_{q}\right)(s, \cdot) \longmapsto h_{i}\left(\varphi_{1}(s, \cdot), \ldots, \varphi_{q}(s, \cdot)\right), \quad i=1,2 .
\end{aligned}
$$

A change of variable $v=w^{\prime}$ transforms the second order equation (2.2) into the following first order system of ordinary differential equations with impulses, delays, and nonlocal conditions in the space $Z^{1 / 2}$.

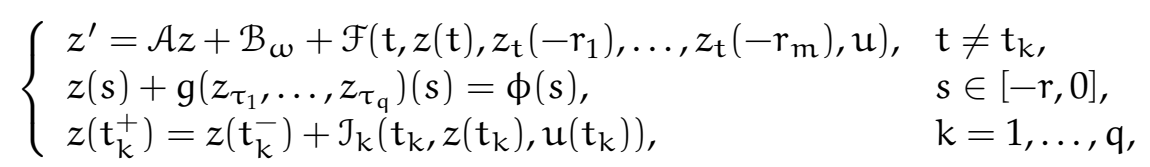

where $u \in C([0, \tau], U), z=(w, v)^{\top}, \phi=\left(\phi_{1}, \phi_{2}\right)^{\top} \in \mathrm{C}([-r, 0], X), z_{\mathrm{t}}$ defined as a function from $[-r, 0]$ to $\mathrm{Z}^{1 / 2}$ by $z_{\mathrm{t}}(\mathrm{s})=z(\mathrm{t}+\mathrm{s}),-\mathrm{r} \leqslant \mathrm{s} \leqslant 0$,

$$
\mathcal{A}=\left[\begin{array}{cc}
0 & \mathrm{I}_{\mathrm{X}} \\
-\gamma \mathrm{A} & -\eta A^{1 / 2}
\end{array}\right]
$$

is an unbounded linear operator with domain $\mathrm{D}(\mathcal{A})=\mathrm{D}(A) \times \mathrm{D}\left(\mathrm{A}^{1 / 2}\right)$, IX represents the identity in $\mathrm{X}$,

$$
\begin{aligned}
\mathcal{B}_{\omega}: \mathrm{u} & \longrightarrow \mathrm{Z}^{1 / 2}, \\
\mathrm{u} & \longmapsto\left(0, \mathrm{~B}_{\omega} \mathrm{u}\right)^{\top}, \\
\mathcal{J}_{\mathrm{k}}:[0, \tau] \times \mathrm{Z}^{1 / 2} \times \mathrm{u} & \longrightarrow \mathrm{Z}^{1 / 2}, \\
(\mathrm{t}, \mathrm{z}, \mathrm{u}) & \longmapsto\left(0, \mathrm{I}_{\mathrm{e}}^{\mathrm{e}}(\mathrm{t}, w, v, \mathrm{u})\right)^{\top},
\end{aligned}
$$

$\mathcal{F}:[0, \tau] \times \mathrm{Z}^{1 / 2} \times \mathrm{C}^{\mathrm{m}}\left([-\mathrm{r}, 0], \mathrm{Z}^{1 / 2}\right) \times \mathrm{U} \longrightarrow \mathrm{Z}^{1 / 2}$,

$$
\left(t, z, \phi^{1}, \ldots, \phi^{m}, u\right) \longmapsto\left(f^{e}\left(t, w, v, \phi_{1}^{1}\left(-r_{1}\right), \ldots, \phi_{1}^{m}\left(-r_{m}\right), \phi_{2}^{1}\left(-r_{1}\right), \ldots, \phi_{2}^{m}\left(-r_{m}\right), u\right),\right.
$$

and

$$
\begin{gathered}
g: C^{\mathrm{q}}([-r, 0] ; X) \longrightarrow \mathrm{C}([-r, 0] ; X) \times C([-r, 0] ; X), \\
g\left(\phi^{1}, \ldots, \phi^{\mathrm{q}}\right)(s, \cdot) \longmapsto\left(\begin{array}{l}
g_{1}\left(\phi_{1}^{1}(s, \cdot), \ldots, \phi_{1}^{\mathrm{q}}(s, \cdot)\right. \\
g_{2}\left(\phi_{2}^{1}(s, \cdot), \ldots, \phi_{2}^{\mathrm{q}}(s, \cdot)\right.
\end{array}\right) .
\end{gathered}
$$

Definition 2.2 (Approximate controllability). The system (2.3) is said to be approximately controllable on $[0, \tau]$ if for every $\phi=\left(\phi_{1}, \phi_{2}\right) \in C\left([-r, 0] ; Z^{1 / 2}\right), z^{1} \in Z^{1 / 2}$ and $\varepsilon>0$, there exists $u \in C\left([0, \tau] ; L^{2}(\Omega)\right)$ such that the solution $z(t)$ of $(1.1)$ corresponding to $u$ verifies:

$$
z(0)+\left(h\left(z_{\tau_{1}}, \ldots, z_{\tau_{q}}\right)\right)(0)=\phi(0) \quad \text { and } \quad\left\|z(\tau)-z^{1}\right\|_{Z^{1 / 2}}<\varepsilon
$$

The hypotheses (1.2), (1.3), (1.4), and (1.5), together with the continuous inclusion $X^{1 / 2} \subset X$, yield the following. 
Proposition 2.3. The functions $\mathcal{F}, \mathcal{J}_{k}$, and $\mathrm{g}$ satisfy:

$$
\begin{aligned}
\left\|\mathcal{F}\left(t, z, \phi_{1}, \ldots, \phi_{m}, u\right)\right\|_{Z^{1 / 2}} & \leqslant \bar{a}_{0}\|z\|_{Z^{1 / 2}}^{\alpha_{0}}+\sum_{l=1}^{m} \bar{a}_{l}\left\|\phi_{l}\left(-r_{l}\right)\right\|^{\rho_{l}}+\bar{b}_{0}\|u\|_{u}^{\beta_{0}}+\bar{c}_{0}, \\
\left\|\mathcal{J}_{k}(t, z, u)\right\|_{Z^{1 / 2}} & \leqslant \bar{a}_{k}\|z\|_{Z^{1 / 2}}^{\alpha_{k}}+\bar{b}_{k}\|u\|_{u}^{\beta_{k}}+\bar{c}_{k^{\prime}}, \quad k=1, \ldots, p, \\
\left\|g\left(\phi_{1}, \ldots, \phi_{q}\right)\right\|_{C} & \leqslant \sum_{i=1}^{q} \bar{e}_{i}\left\|\phi_{i}\right\|_{C}^{\zeta_{i}}+\bar{e}_{0}, \quad \phi_{i} \in C \\
\left|g\left(z_{\tau_{1}}, \ldots, z_{\tau_{q}}\right)-g\left(w_{\tau_{1}}, \ldots, w_{\tau_{q}}\right)\right| & \leqslant K\|z-w\|_{P C_{t_{1} \ldots t_{p}}\left([-r, \tau] ; Z^{1 / 2}\right)}, \quad z, w \in P C_{t_{1} \ldots t_{p}} .
\end{aligned}
$$

It is well known that the operator $\mathcal{A}$ generates a strongly continuous semigroup $\{\mathbf{T}(\mathbf{t})\}_{\mathrm{t} \geqslant 0}$ in the space $\mathrm{Z}^{1 / 2}$, which is also analytic. Furthermore, Lemma 2.1 in [14] yields the following.

Proposition 2.4. The semigroup $\{\mathrm{T}(\mathrm{t})\}_{\mathrm{t} \geqslant 0}$ generated by the operator $\mathcal{A}$ is compact and has the following representation

$$
\mathrm{T}(\mathrm{t}) z=\sum_{j=1}^{\infty} e^{\mathrm{A}_{j} \mathrm{t}} \mathrm{P}, z, \quad z \in \mathrm{Z}^{1 / 2}, \quad \mathrm{t} \geqslant 0,
$$

where $\left\{\mathrm{P}_{\mathbf{j}}\right\}_{j} \geqslant 0$ is a complete family of orthogonal projections in the Hilbert space $\mathrm{Z}^{1 / 2}$ given by (2.1) and

$$
A_{j}=R_{j} P_{j}, \quad R_{j}=\left[\begin{array}{cc}
0 & 1 \\
-\gamma \lambda_{j} & -\eta \lambda_{j}^{1 / 2}
\end{array}\right], \quad j \geqslant 1
$$

Moreover, $e^{A_{j} t}=e^{R_{j} t} P_{j}$, the eigenvalues of $R_{j}$ are

$$
\lambda=-\lambda_{j}^{1 / 2}\left(\frac{\eta \pm \sqrt{\eta^{2}-4 \gamma}}{2}\right), \quad j=1,2, \ldots, \quad \text { and } \quad A_{j}^{*}=R_{j} P_{j}, \quad R_{j}^{*}=\left[\begin{array}{cc}
0 & -1 \\
\gamma \lambda_{j} & -\eta \lambda_{j}^{1 / 2}
\end{array}\right],
$$

and

$$
\|T(t)\| \leqslant M(\eta, \gamma) e^{-\beta t}, \quad t \geqslant 0,
$$

where

$$
\beta=\lambda_{1}^{\frac{1}{2}} \min \left\{\operatorname{Re}\left(\frac{\eta \pm \sqrt{\eta^{2}-4 \gamma}}{2}\right)\right\}
$$

\section{Controllability of the unperturbed linear equation}

In this section, we shall present some characterization of the interior approximate controllability of the linear strongly damped equation without impulses, delays, and nonlocal conditions. To this end, we note that, for all $z_{0} \in \mathrm{Z}^{1 / 2}$ and $u \in \mathrm{L}_{2}([0, \tau] ; \mathrm{U})$ the initial value problem

$$
\left\{\begin{array}{l}
z^{\prime}=\mathcal{A} z+\mathcal{B}_{\omega} u(t), \quad z \in Z \\
z(0)=z_{0}
\end{array}\right.
$$

where the control function $u$ belongs to $\mathrm{L}_{2}([0, \tau] ; \mathrm{U})$, admits only one mild solution given by

$$
z(t)=T(t) z_{0}+\int_{0}^{t} T(t-s) \mathcal{B}_{\omega} u(s) d s, \quad t \in[0, \tau]
$$


Definition 3.1. For system (3.1) we define the following linear operators. The controllability operator (for $\tau>0) \mathcal{G}: \mathrm{L}_{2}([0, \tau] ; \mathrm{U}) \longrightarrow \mathrm{Z}^{1 / 2}$ is given by

$$
\mathcal{G} u=\int_{0}^{\tau} \mathrm{T}(\tau-\mathrm{s}) \mathcal{B}_{\omega} u(s) d s,
$$

whose adjoint operator $\mathcal{G}^{*}: Z^{1 / 2} \longrightarrow \mathrm{L}_{2}([0, \tau] ; Z)$ is given by

$$
\left(\mathcal{G}^{*} z\right)(s)=\mathcal{B}_{\omega}^{*} T^{*}(\tau-s) z, \quad \forall s \in[0, \tau], \quad \forall z \in Z^{1 / 2} .
$$

Therefore, the Grammian operator $\mathcal{W}: Z^{1 / 2} \rightarrow Z^{1 / 2}$ is given by

$$
\mathcal{W} z=\mathcal{G G}^{*} z=\int_{0}^{\tau} \mathrm{T}(\tau-\mathrm{s}) \mathcal{B}_{\omega} \mathcal{B}_{\omega}^{*} \mathrm{~T}^{*}(\tau-\mathrm{s}) \mathrm{ds} .
$$

The following lemma holds in general for a linear bounded operator $\mathcal{G}: W \rightarrow Z$ between Hilbert spaces $W$ and $Z$.

Lemma $3.2([2,6,7,17])$. The equation (3.1) is approximately controllable on $[0, \tau]$ if, and only if, one of the following statements holds:

a) $\overline{\operatorname{Rang}(\mathcal{G})}=Z^{1 / 2}$;

b) $\operatorname{Ker}\left(\mathcal{G}^{*}\right)=\{0\}$;

c) $\left\langle\mathcal{G G}^{*} z, z\right\rangle>0, z \neq 0$ in $Z^{1 / 2}$;

d) $\lim _{\alpha \rightarrow 0^{+}} \alpha\left(\alpha \mathrm{I}+\mathcal{G G}^{*}\right)^{-1} z=0$;

e) $\mathcal{B}_{\omega}^{*} T^{*}(\mathrm{t}) z=0, \quad \forall \mathrm{t} \in[0, \tau], \quad \Rightarrow z=0$.

Remark 3.3. Lemma 3.2 implies that for all $z \in Z^{1 / 2}$, we have that $\mathcal{G} u_{\alpha}=z-\alpha\left(\alpha I+\mathcal{G} \mathcal{G}^{*}\right)^{-1} z$, where

$$
\mathrm{u}_{\alpha}=\mathcal{G}^{*}\left(\alpha \mathrm{I}+\mathcal{G G}^{*}\right)^{-1} z, \quad \alpha \in(0,1] .
$$

So, $\lim _{\alpha \rightarrow 0} \mathcal{G} \mathrm{u}_{\alpha}=z$ and the error $\mathrm{E}_{\alpha} z$ of this approximation is given by

$$
\mathrm{E}_{\alpha} z=\alpha\left(\alpha \mathrm{I}+\mathcal{G G}^{*}\right)^{-1} z, \quad \alpha \in(0,1] .
$$

Also, Lemma 3.2 implies that the family of linear operators $\Gamma_{\alpha}: Z \rightarrow L_{2}(0, \tau ; U)$, defined for $0<\alpha \leqslant 1$ by

$$
\Gamma_{\alpha} z=\mathcal{B}_{\omega}^{*} T^{*}(\cdot)\left(\alpha \mathrm{I}+\mathcal{G} \mathcal{G}^{*}\right)^{-1} z=\mathcal{G}^{*}\left(\alpha \mathrm{I}+\mathcal{G G}^{*}\right)^{-1} z,
$$

satisfies

$$
\lim _{\alpha \rightarrow 0} \mathcal{G} \Gamma_{\alpha}=\mathrm{I},
$$

in the strong topology.

Proposition 3.4 ([17]). If $\overline{\operatorname{Rang}(\mathcal{G})}=\mathrm{Z}^{1 / 2}$, then

$$
\sup _{\alpha>0}\left\|\alpha\left(\alpha \mathrm{I}+\mathcal{G G}^{*}\right)^{-1}\right\| \leqslant 1
$$

Remark 3.5. The proof of the following Theorem follows from the foregoing characterization of dense range linear operators and the classical unique continuation for elliptic equations (see [23]), and it is similar to the one given in Theorem 4.1 in [16].

Theorem 3.6. Under the conditions mentioned above, system (3.1) is approximately controllable on [0, $\tau]$. Moreover, a sequence of controls steering the system (3.1) from initial state $z_{0}$ to an $\in$ neighborhood of the final state $z_{1}$ at time $\tau>0$ is given by

$$
u_{\alpha}(t)=\mathcal{B}_{\omega}^{*} T^{*}(\tau-t)\left(\alpha \mathrm{I}+\mathcal{G G}^{*}\right)^{-1}\left(z_{1}-\mathrm{T}(\tau) z_{0}\right),
$$

and the error of this approximation $\mathrm{E}_{\alpha}$ is given by

$$
\mathrm{E}_{\alpha}=\alpha\left(\alpha \mathrm{I}+\mathcal{G G}^{*}\right)^{-1}\left(z_{1}-\mathrm{T}(\tau) z_{0}\right) .
$$




\section{The system with impulses, delays, and nonlocal conditions}

In this section, we shall prove the main result of this paper, the interior approximate controllability of the semilinear strongly damped wave equation with impulses, delays, and nonlocal conditions given by (1.1), which is equivalent to prove the approximate controllability of the system (2.3). To this end, for all $\phi \in \mathrm{C}$ and $u \in \mathrm{C}([0, \tau] ; \mathrm{u})$ the initial value problem, according with the recent work from $[15,19,27]$,

$$
\begin{cases}z^{\prime}=\mathcal{A} z+\mathcal{B}_{\omega} u+\mathcal{F}\left(\mathrm{t}, z(\mathrm{t}), z_{\mathrm{t}}\left(-\mathrm{r}_{1}\right), z_{\mathrm{t}}\left(-\mathrm{r}_{2}\right), \ldots, z_{\mathrm{t}}\left(-\mathrm{r}_{\mathrm{m}}\right), \mathrm{u}\right), & \mathrm{t} \in(0, \tau], \\ z(\mathrm{~s})+\left(\mathrm{g}\left(z_{\tau_{1}}, z_{\tau_{2}}, \ldots, z_{\tau_{\mathrm{q}}}\right)\right)(\mathrm{s})=\phi(s), & s \in[-r, 0], \\ z\left(\mathrm{t}_{\mathrm{k}}^{+}\right)=z\left(\mathrm{t}_{\mathrm{k}}^{-}\right)+\mathcal{J}_{\mathrm{k}}\left(\mathrm{t}_{\mathrm{k}}, z\left(\mathrm{t}_{\mathrm{k}}\right), \mathrm{u}\left(\mathrm{t}_{\mathrm{k}}\right)\right), & \mathrm{k}=1,2,3, \ldots, \mathrm{p},\end{cases}
$$

admits only one mild solution $z \in \mathrm{PC}_{\mathrm{t}_{1} \cdots \mathrm{t}_{\mathrm{P}}}\left([-\mathrm{r}, \tau] ; \mathrm{Z}^{1 / 2}\right)$ given by

$$
\begin{aligned}
z(t)= & T(t) \phi(0)-T(t)\left[\left(g\left(z_{t_{1}}, \ldots, z_{t_{q}}\right)\right)(0)\right]+\int_{0}^{t} T(t-s) \mathcal{B}_{\omega} u(s) d s \\
& +\int_{0}^{t} T(t-s) \mathcal{F}\left(s, z(s), z_{s}\left(-r_{1}\right), z_{s}\left(-r_{2}\right), \ldots, z_{s}\left(-r_{m}\right), u(s)\right) d s \\
& +\sum_{0<t_{k}<t} T\left(t-t_{k}\right) \mathcal{J}_{k}\left(t_{k}, z\left(t_{k}\right), u\left(t_{k}\right)\right), \quad t \in[0, \tau], \\
z(t) & +\left(g\left(z_{\tau_{1}}, \ldots, z_{\tau_{q}}\right)\right)(t)=\phi(t), \quad t \in[-r, 0],
\end{aligned}
$$

according to $[4,11,15]$.

Now, we are ready to present and prove the main result of this paper, which is the interior approximate controllability of the semilinear strongly damped wave equation with impulses, delays, and nonlocal conditions (1.1). Note that, we shall define the following operator

$$
\mathcal{S}^{\alpha}: P C_{t_{1} \cdots t_{P}}\left([-r, \tau] ; Z^{1 / 2}\right) \times C([0, \tau] ; u) \rightarrow P_{t_{1} \cdots t_{p}}\left([-r, \tau] ; Z^{1 / 2}\right) \times C([0, \tau] ; u)
$$

by the following formula:

$$
(y, v)=\left(\mathcal{S}_{1}^{\alpha}(z, u), \mathcal{S}_{2}^{\alpha}(z, u)\right)=\mathcal{S}^{\alpha}(z, u),
$$

where

$$
\begin{aligned}
y(t)= & \mathcal{S}_{1}^{\alpha}(z, u)(t)=T(t) \phi(0)-T(t)\left[\left(g\left(z_{t_{1}}, \ldots, z_{t_{q}}\right)\right)(0)\right] \\
& +\int_{0}^{t} T(t-s) \mathcal{B}_{\omega}\left(\Gamma_{\alpha} \mathcal{L}(z, u)\right)(s) d s \\
& +\int_{0}^{t} T(t-s) \mathcal{F}\left(s, z(s), z\left(s-r_{1}\right), z\left(s-r_{2}\right), \ldots, z\left(s-r_{m}\right), u(s)\right) d s \\
& +\sum_{0<t_{k}<t} T\left(t-t_{k}\right) \mathcal{J}_{k}\left(t_{k}, z\left(t_{k}\right), u\left(t_{k}\right)\right), t \in[0, \tau], \\
y(t) & +\left(g\left(z_{t_{1}}, \ldots, z_{t_{q}}\right)\right)(t)=\phi(t), \quad t \in[-r, 0],
\end{aligned}
$$

and

$$
v(t)=\mathcal{S}_{2}^{\alpha}(z, u)(t)=\left(\Gamma_{\alpha} \mathcal{L}(z, u)\right)(t)=\mathcal{B}_{\omega}^{*} T^{*}(\tau-t)(\alpha I+\mathcal{W})^{-1} \mathcal{L}(z, u), t \in[0, \tau],
$$

with $\mathcal{L}: \mathrm{PCPC}_{\mathrm{t}_{1} \cdots \mathrm{t}_{\mathrm{P}}}\left([-\mathrm{r}, \tau] ; \mathrm{Z}^{1 / 2}\right) \times \mathrm{C}([0, \tau] ; \mathrm{U}) \rightarrow \mathrm{Z}^{1 / 2}$ given by

$$
\begin{aligned}
\mathcal{L}(z, \mathrm{u})= & z_{1}-\mathrm{T}(\tau) \phi(0)+\mathrm{T}(\tau)\left[\left(\mathrm{g}\left(z_{\tau_{1}}, \ldots, z_{\tau_{\mathrm{q}}}\right)\right)(0)\right] \\
& -\int_{0}^{\tau} \mathrm{T}(\tau-s) \mathcal{F}\left(s, z(s), z\left(s-r_{1}\right), \ldots, z\left(s-r_{\mathrm{m}}\right), \mathrm{u}(\mathrm{s})\right) \mathrm{d} s \\
& -\sum_{0<\mathrm{t}_{k}<\tau} \mathrm{T}\left(\tau-\mathrm{t}_{\mathrm{k}}\right) \mathcal{J}_{k}\left(\mathrm{t}_{\mathrm{k}}, z\left(\mathrm{t}_{\mathrm{k}}\right), \mathrm{u}\left(\mathrm{t}_{\mathrm{k}}\right)\right) .
\end{aligned}
$$


Theorem 4.1. Under conditions (1.2)-(1.5), the nonlinear system (1.1) is approximately controllable on $[0, \tau]$. Moreover, a sequence of controls steering the system (1.1) from initial state $\phi$ to an $\epsilon$-neighborhood of the final state $z^{1}$ at time $\tau>0$ is given by

$$
\mathrm{u}_{\alpha}(\mathrm{t})=\mathcal{B}_{\omega}^{*} \mathrm{~T}^{*}(\tau-\mathrm{t})(\alpha \mathrm{I}+\mathcal{W})^{-1} \mathcal{L}\left(z_{\alpha}, \mathrm{u}_{\alpha}\right), \mathrm{t} \in[0, \tau],
$$

and the error of this approximation $\mathrm{E}_{\alpha} z$ is given by

$$
\begin{aligned}
\mathrm{E}_{\alpha} z= & \alpha(\alpha \mathrm{I}+\mathcal{W})^{-1} \mathcal{L}\left(z^{\alpha}, \mathrm{u}_{\alpha}\right), \\
z^{\alpha}(\mathrm{t})= & \mathrm{T}(\mathrm{t}) \phi(0)-\mathrm{T}(\mathrm{t})\left[\left(\mathrm{g}\left(z_{\tau_{1}}, \ldots, z_{\tau_{\mathrm{q}}}\right)\right)(0)\right]+\int_{0}^{\mathrm{t}} \mathrm{T}(\mathrm{t}-\mathrm{s}) \mathcal{B}_{\omega} \mathrm{u}_{\alpha}(\mathrm{s}) \mathrm{d} s \\
& +\int_{0}^{\mathrm{t}} \mathrm{T}(\mathrm{t}-\mathrm{s}) \mathcal{F}\left(s, z^{\alpha}(s), z^{\alpha}\left(s-r_{1}\right), \ldots, z^{\alpha}\left(s-r_{\mathrm{m}}\right), \mathrm{u}_{\alpha}(\mathrm{s})\right) \mathrm{d} s \\
& +\sum_{0<\mathrm{t}_{\mathrm{k}}<\mathrm{t}} \mathrm{T}\left(\mathrm{t}-\mathrm{t}_{\mathrm{k}}\right) \mathcal{J}_{\mathrm{k}}\left(\mathrm{t}_{\mathrm{k}}, z^{\alpha}\left(\mathrm{t}_{\mathrm{k}}\right), \mathrm{u}_{\alpha}\left(\mathrm{t}_{\mathrm{k}}\right)\right), \mathrm{t} \in[0, \tau], \\
z^{\alpha}(\mathrm{t}) & +\left(\mathrm{g}\left(z_{\mathrm{t}_{1}}, \ldots, z_{\mathrm{t}_{\mathrm{q}}}\right)\right)(\mathrm{t})=\phi(\mathrm{t}), \quad \mathrm{t} \in[-r, 0] .
\end{aligned}
$$

Proof. We shall prove this Theorem by claims. Before proving the Theorem, we note that $\left\|B_{\omega}\right\|=1$ and $\|T(t)\| \leqslant M(\eta, \gamma) e^{-\beta t}, \quad t \geqslant 0$.

Claim 1. The operator $\mathcal{S}^{\alpha}$ is continuous. In fact, it is enough to prove that the operators:

$$
\mathcal{S}_{1}^{\alpha}: P C_{t_{1} \cdots t_{P}}\left([-r, \tau] ; Z^{1 / 2}\right) \times C([0, \tau] ; U) \rightarrow P C_{t_{1} \cdots t_{P}}\left([-r, \tau] ; Z^{1 / 2}\right)
$$

and

$$
\mathcal{S}_{2}^{\alpha}: \mathrm{PC}_{\mathrm{t}_{1} \cdots \mathrm{t}_{\mathrm{p}}}\left([-\mathrm{r}, \tau] ; \mathrm{Z}^{1 / 2}\right) \times \mathrm{C}([0, \tau] ; \mathrm{U}) \rightarrow \mathrm{C}([0, \tau] ; \mathrm{U}),
$$

defined above are continuous. The continuity of $\mathcal{S}_{1}^{\alpha}$ follows from the continuity of the nonlinear functions $g, \mathcal{F}(t, \phi, u), J_{k}(t, z, u)$ and the following estimate

$$
\begin{aligned}
& \left\|\mathcal{S}_{1}^{\alpha}(z, u)(t)-\mathcal{S}_{1}^{\alpha}(w, v)(t)\right\| \\
& \leqslant \tilde{\mathrm{K}}\|z-w\|_{\mathrm{PC}_{\mathrm{t}_{1} \cdots \mathrm{t}_{\mathrm{p}}}\left([-r, \tau] ; \mathrm{Z}^{1 / 2}\right)}+\int_{0}^{\mathrm{t}} \mathrm{M}(\eta, \gamma) e^{-\beta(\mathrm{t}-\mathrm{s})}\left\|(\alpha \mathrm{I}+\mathcal{W})^{-1}\right\|\|\mathcal{L}(z, \mathrm{u})-\mathcal{L}(w, v)\| \mathrm{d} s \\
& +\int_{0}^{t} M(\eta, \gamma) e^{-\beta(t-s)} \| \mathcal{F}\left(s, z(s), z\left(s-r_{1}\right), \ldots, z\left(s-r_{m}\right), u(s)\right) \\
& -\mathcal{F}\left(s, w(s), w\left(s-r_{1}\right), \ldots, w\left(s-r_{m}\right), v(s)\right) \| d s \\
& +\sum_{0<t_{k}<t} M(\eta, \gamma) e^{-\beta\left(t-t_{k}\right)}\left\|\mathcal{J}_{k}\left(t_{k}, z\left(t_{k}\right), u\left(t_{k}\right)\right)-\mathcal{J}_{k}\left(t_{k}, w\left(t_{k}\right), v\left(t_{k}\right)\right)\right\| .
\end{aligned}
$$

On the other hand,

$$
\begin{aligned}
& \|\mathcal{L}(z, u)-\mathcal{L}(w, v)\| \\
& \quad \leqslant \tilde{\mathrm{K}}\|z-w\|_{\mathrm{PC}_{\mathrm{t}_{1} \cdots \mathrm{t}_{\mathrm{p}}\left([-r, \tau] ; \mathrm{Z}^{1 / 2}\right)}+\tau M(\eta, \gamma) \sup _{s \in[0, \tau]}\|\mathcal{F}(s, z(s), \ldots, \mathrm{u}(\mathrm{s}))-\mathcal{F}(s, w(s), \ldots, v(s))\|} \\
& \quad+\sum_{0<\mathrm{t}_{\mathrm{k}}<\tau} M(\eta, \gamma) e^{-\beta\left(\tau-\mathrm{t}_{\mathrm{k}}\right)}\left\|\mathcal{J}_{\mathrm{k}}\left(\mathrm{t}_{\mathrm{k}}, z\left(\mathrm{t}_{\mathrm{k}}\right), \mathrm{u}\left(\mathrm{t}_{\mathrm{k}}\right)\right)-\mathcal{J}_{\mathrm{k}}\left(\mathrm{t}_{\mathrm{k}}, w\left(\mathrm{t}_{\mathrm{k}}\right), v\left(\mathrm{t}_{\mathrm{k}}\right)\right)\right\| .
\end{aligned}
$$

Therefore

$$
\begin{aligned}
\left\|\mathcal{S}_{2}^{\alpha}(z, \mathrm{u})-\mathcal{S}_{2}^{\alpha}(w, v)\right\| \leqslant \tilde{\mathrm{K}}\|z-w\|_{\mathrm{PC}_{\mathrm{t}_{1} \cdots \mathrm{t}_{\mathrm{p}}}\left([-\mathrm{r}, \tau] ; \mathrm{Z}^{1 / 2}\right)} & +\mathrm{L}_{1} \sup _{\mathrm{s} \in[0, \tau]}\|\mathcal{F}(\mathrm{s}, z(\mathrm{~s}), \ldots, \mathrm{u}(\mathrm{s}))-\mathcal{F}(\mathrm{s}, \mathfrak{w}(\mathrm{s}), \ldots, v(\mathrm{~s}))\|
\end{aligned}
$$




$$
+\mathrm{L}_{2} \sum_{0<\mathrm{t}_{\mathrm{k}}<\tau}\left\|\mathcal{J}_{\mathrm{k}}\left(\mathrm{t}_{\mathrm{k}}, z\left(\mathrm{t}_{\mathrm{k}}\right), \mathrm{u}\left(\mathrm{t}_{\mathrm{k}}\right)\right)-\mathrm{J}_{\mathrm{k}}\left(\mathrm{t}_{\mathrm{k}}, w\left(\mathrm{t}_{\mathrm{k}}\right), v\left(\mathrm{t}_{\mathrm{k}}\right)\right)\right\|
$$

where $\mathrm{L}_{1}=\tau M(\eta, \gamma)\left(\tau M(\eta, \gamma)\left\|(\alpha \mathrm{I}+\mathcal{W})^{-1}\right\|+1\right)$ and $\mathrm{L}_{2}=\left(1+\tau M(\eta, \gamma)\left\|(\alpha \mathrm{I}+\mathcal{W})^{-1}\right\|\right)$. The continuity of the operator $\mathcal{S}_{2}^{\alpha}$ follows from the continuity of the operators $\mathcal{L}$ and $\Gamma_{\alpha}$ define above.

Claim 2. The operator $\mathcal{S}^{\alpha}$ is compact. In fact, let $\mathrm{D}$ be a bounded subset of $\mathrm{PC}_{\mathrm{t}_{1} \cdots \mathrm{t}_{\mathrm{p}}}\left(\mathrm{J} ; \mathrm{Z}^{1 / 2}\right) \times \mathrm{C}(\mathrm{J} ; \mathrm{U})$. It follows that $\forall(z, u) \in D$, we have

$$
\|\mathcal{F}(\cdot, z, u)\| \leqslant \mathrm{L}_{3}, \quad\left\|(\alpha \mathrm{I}+\mathcal{W})^{-1} \mathcal{L}(z, u)\right\| \leqslant \mathrm{L}_{4}, \quad\|\mathcal{L}(z, \mathrm{u})\| \leqslant \mathrm{L}_{5}, \quad\left\|\mathcal{J}_{k}(\cdot, z, \mathrm{u})\right\| \leqslant l_{k}, \quad k=1,2, \ldots, p .
$$

Therefore, $\mathcal{S}^{\alpha}(\mathrm{D})$ is uniformly bounded.

Now, consider the following estimate

$$
\left\|\mathcal{S}^{\alpha}(z, \mathfrak{u})\left(\sigma_{2}\right)-\mathcal{S}^{\alpha}(z, \mathfrak{u})\left(\sigma_{1}\right)\right\|\|=\| \mathcal{S}_{1}^{\alpha}(z, u)\left(\sigma_{2}\right)-\mathcal{S}_{1}^{\alpha}(z, u)\left(\sigma_{1}\right)\|+\| \mathcal{S}_{2}^{\alpha}(z, u)\left(\sigma_{2}\right)-\mathcal{S}_{2}^{\alpha}(z, u)\left(\sigma_{1}\right) \| .
$$

Without lose of generality, we assume that $0<\sigma_{1}<\sigma_{2}$. On the other hand, we have that:

$$
\begin{aligned}
& \left\|\mathcal{S}_{1}^{\alpha}(z, u)\left(\sigma_{2}\right)-\mathcal{S}_{1}^{\alpha}(z, u)\left(\sigma_{1}\right)\right\| \leqslant\left\|\mathrm{T}\left(\sigma_{2}\right)-\mathrm{T}\left(\sigma_{1}\right)\right\|\left\{\|\phi\|+\left\|\left[\left(g\left(z_{\tau_{1}}, \ldots, z_{\tau_{\mathrm{q}}}\right)\right)(0)\right]\right\|\right\} \\
& +\int_{0}^{\sigma_{1}}\left\|\mathrm{~T}\left(\sigma_{2}-s\right)-\mathrm{T}\left(\sigma_{1}-\mathrm{s}\right)\right\|\|\mathcal{L}(z, \mathrm{u})(\mathrm{s})\| \mathrm{d} s \\
& +\int_{\sigma_{1}}^{\sigma_{2}}\left\|\mathrm{~T}\left(\sigma_{2}-s\right)\right\|\|\mathcal{L}(z, u)(s)\| \mathrm{d} s \\
& +\int_{0}^{\sigma_{1}}\left\|\mathrm{~T}\left(\sigma_{2}-s\right)-\mathrm{T}\left(\sigma_{1}-s\right)\right\|\left\|\mathcal{F}\left(s, z(s), z\left(s-r_{1}\right), \ldots, z\left(s-r_{m}\right) u(s)\right)\right\| \mathrm{d} s \\
& +\int_{\sigma_{1}}^{\sigma_{2}}\left\|\mathrm{~T}\left(\sigma_{2}-s\right)\right\|\left\|\mathcal{F}\left(s, z(s), z\left(s-r_{1}\right), \ldots, z\left(s-r_{m}\right) u(s)\right)\right\| d s \\
& +\sum_{0<t_{k}<\sigma_{1}}\left\|\mathrm{~T}\left(\sigma_{2}-t_{k}\right)-\mathrm{T}\left(\sigma_{1}-t_{k}\right)\right\|\left\|\mathcal{J}_{k}\left(t_{k}, z\left(t_{k}\right), u\left(t_{k}\right)\right)\right\| \\
& +\sum_{\sigma_{1}<t_{k}<\sigma_{2}}\left\|T\left(\sigma_{2}-t_{k}\right) \mathcal{J}_{k}\left(t_{k}, z\left(t_{k}\right), u\left(t_{k}\right)\right)\right\|,
\end{aligned}
$$

and

$$
\left\|\mathcal{S}_{2}^{\alpha}(z, u)\left(\sigma_{2}\right)-\mathcal{S}_{2}^{\alpha}(z, u)\left(\sigma_{1}\right)\right\| \leqslant\left\|T^{*}\left(\tau-\sigma_{2}\right)-T^{*}\left(\tau-\sigma_{1}\right)\right\|\left\|(\alpha \mathrm{I}+\mathcal{W})^{-1} \mathcal{L}(z, u)\right\|
$$

On the other hand, since $T(t)$ is a compact operator for $t>0$, then from [22] we know that the function $0<\mathrm{t} \rightarrow \mathrm{T}(\mathrm{t})$ is uniformly continuous. So,

$$
\lim _{\left|\sigma_{2}-\sigma_{1}\right| \rightarrow 0}\left\|\mathrm{~T}\left(\sigma_{2}\right)-\mathrm{T}\left(\sigma_{1}\right)\right\|=0 .
$$

Consequently, if we take a sequence $\left\{\varphi_{j}: j=1,2, \ldots\right\}$ on $\mathcal{S}^{\alpha}(D)$, this sequence is uniformly bounded and equicontinuous on the interval $\left[-r, t_{1}\right]$ and, by Arzela theorem, there is a sub-sequence $\left\{\varphi_{j}^{1}: j=1,2, \ldots\right\}$ of $\left\{\varphi_{j}: j=1,2, \ldots\right\}$, which is uniformly convergent on $\left[-r, t_{1}\right]$.

Consider the sequence $\left\{\varphi_{j}^{1}: j=1,2, \ldots\right\}$ on the interval $\left(t_{1}, t_{2}\right]$. On this interval the sequence $\left\{\varphi_{j}^{1}: j=\right.$ $1,2, \ldots\}$ is uniformly bounded and equicontinuous, and for the same reason, it has a sub-sequence $\left\{\varphi_{j}^{2}\right\}$ uniformly convergent on $\left[-r, t_{2}\right]$.

Continuing this process for the intervals $\left(t_{2}, t_{3}\right],\left(t_{3}, t_{4}\right], \ldots,\left(t_{p}, \tau\right]$, we see that the sequence $\left\{\varphi_{j}^{p+1}\right.$ : $j=1,2, \ldots\}$ converges uniformly on the interval $[-r, \tau]$. This means that $\overline{\mathcal{S}^{\alpha}(D)}$ is compact, which implies the operator $\mathcal{S}^{\alpha}$ is compact. 


\section{Claim 3.}

$$
\lim _{\||(z, u) \|| \rightarrow \infty} \frac{\left\|\mathcal{S}^{\alpha}(z, u)\right\| \mid}{\||(z, \mathfrak{u}) \||}=0,
$$

where $\left\|\left|(z, u)\|\mid=\| z\|+\| u \|\right.\right.$ is the norm in the space $\mathrm{PC}_{t_{1} \cdots t_{p}}\left([-r, \tau] ; Z^{1 / 2}\right) \times C\left(0, \tau ; Z^{1 / 2}\right)$. In fact, consider the following estimates:

$$
\begin{aligned}
\|\mathcal{L}(z, \mathrm{u})\| \leqslant & \mathrm{M}_{1}+\mathrm{M}_{2}\left\{\overline{\mathrm{a}}_{0}\|z\|^{\alpha_{0}}+\sum_{\mathrm{l}=1}^{\mathrm{m}} \overline{\mathrm{a}}_{\mathrm{l}}|z|^{\rho_{\mathrm{l}}}+\sum_{i=1}^{\mathrm{q}} \overline{\mathrm{e}}_{\mathrm{i}}|z|^{\zeta_{i}}+\overline{\mathrm{b}}_{0}\|\mathrm{u}\|^{\beta_{0}}+\overline{\mathrm{c}}_{0}\right\} \\
& +\mathrm{M}_{3} \sum_{0<\mathrm{t}_{\mathrm{k}}<\tau}\left\{\overline{\mathrm{a}}_{\mathrm{k}}\|z\|^{\alpha_{\mathrm{k}}}+\overline{\mathrm{b}}_{\mathrm{k}}\|\mathrm{u}\|^{\beta_{\mathrm{k}}}+\overline{\mathrm{c}}_{\mathrm{k}}\right\},
\end{aligned}
$$

where

$$
\begin{aligned}
M_{1}= & \left\|z_{1}\right\|+\left(M(\eta, \gamma) e^{-\beta \tau}\|\phi(0)\|, \quad M_{2}=\frac{1}{-\beta}\left(M(\eta, \gamma) e^{-\beta \tau}-1\right), \quad \text { and } \quad M_{3}=M(\eta, \gamma) e^{-\beta \tau}\right. \\
\left\|\mathcal{S}_{2}^{\alpha}(z, u)\right\| \leqslant & M_{3} M_{1}\left\|(\alpha I+\mathcal{W})^{-1}\right\|+M_{3} M_{2}\left\|(\alpha I+\mathcal{W})^{-1}\right\|\left\{\bar{a}_{0}\|z\|^{\alpha_{0}}+\sum_{l=1}^{m} \bar{a}_{l}|z|^{\rho_{l}}+\sum_{i=1}^{q} \bar{e}_{i}|z|^{\zeta_{i}}+\bar{b}_{0}\|u\|^{\beta_{0}}+\bar{c}_{0}\right\} \\
& +M_{3} M_{2}\left\|(\alpha I+\mathcal{W})^{-1}\right\| \sum_{0<t_{k}<\tau}\left\{\bar{a}_{k}\|z\|^{\alpha_{k}}+\bar{b}_{k}\|u\|^{\beta_{k}}+\bar{c}_{k}\right\},
\end{aligned}
$$

and

$$
\begin{aligned}
\left\|\mathcal{S}_{1}^{\alpha}(z, u)\right\| \leqslant & M_{3}\left\{\left\|z^{0}\right\|+M_{1} M_{2}\left\|(\alpha I+\mathcal{W})^{-1}\right\|\right\} \\
& \left.+M_{2}\left\{1+M_{2} M_{3}\left\|(\alpha I+\mathcal{W})^{-1}\right\|\right\} \bar{a}_{0}\|z\|^{\alpha_{0}}+\sum_{l=1}^{m} \bar{a}_{l}|z|^{\rho_{l}}+\sum_{i=1}^{q} \bar{e}_{i}|z|^{\zeta_{i}}+\bar{b}_{0}\|u\|^{\beta_{0}}+c_{0}\right\} \\
& +M_{3}\left\{1+M_{2} M_{3}\left\|(\alpha I+\mathcal{W})^{-1}\right\|\right\} \sum_{0<t_{k}<\tau}\left\{\bar{a}_{k}\|z\|^{\alpha_{k}}+\bar{b}_{k}\|u\|^{\beta_{k}}+\bar{c}_{k}\right\} .
\end{aligned}
$$

Therefore,

$$
\begin{aligned}
\left\|\mathcal{S}^{\alpha}(z, u)\right\| l= & \left\|\mathcal{S}_{1}^{\alpha}(z, u)\right\|+\left\|\mathcal{S}_{2}^{\alpha}(z, u)\right\| \\
\leqslant & M_{4}+\left\{M_{3} M_{2}\left\|(\alpha \mathrm{I}+\mathcal{W})^{-1}\right\|\left\{1+2 M_{2}\right\}\left\{\bar{a}_{0}\|z\|^{\alpha_{0}}+\sum_{l=1}^{m} \bar{a}_{l}|z|^{\rho_{l}}+\sum_{i=1}^{q} \bar{e}_{i}|z|^{\zeta_{i}}+\bar{b}_{0}\|u\|^{\beta_{0}}+\bar{c}_{0}\right\}\right. \\
& +\left\{M_{3} M_{2}\left\|(\alpha I+\mathcal{W})^{-1}\right\|\left\{1+M_{3}\right\}+M_{3}\right\} \sum_{0<t_{k}<\tau}\left\{\bar{a}_{k}\|z\|^{\alpha_{k}}+\bar{b}_{k}\|u\|^{\beta_{k}}+\bar{c}_{k}\right\},
\end{aligned}
$$

where $M_{4}$ is given by:

$$
M_{4}=M_{3}\left\{\left\|z^{0}\right\|+\left(M_{2}+1\right) M_{1}\left\|(\alpha I+\mathcal{W})^{-1}\right\|\right\}
$$

Hence,

$$
\begin{aligned}
\frac{\left\|\mathcal{S}^{\alpha}(z, u)\right\| \mid}{\|\|(z, u) \| \mid} \leqslant & \frac{M_{4}}{\|z\|+\|u\|}+\left\{M_{3} M_{2}\left\|(\alpha I+\mathcal{W})^{-1}\right\|\left\{1+M_{2}\right\}\right\} \\
& \times\left\{\bar{a}_{0}\|z\|^{\alpha_{0}-1}+\sum_{l=1}^{m} \bar{a}_{l}|z|^{\rho_{l}-1}+\sum_{i=1}^{\mathrm{q}} \bar{e}_{i}|z|^{\eta_{i}-1}+\bar{b}_{0}\|u\|^{\beta_{0}-1}+\frac{\bar{c}_{0}}{\|z\|+\|u\|}\right\} \\
& +\left\{M_{3} M_{2}\left\|(\alpha I+\mathcal{W})^{-1}\right\|\left\{1+M_{3}\right\}+M_{3}\right\} \times \sum_{0<t_{k}<\tau}\left\{\bar{a}_{k}\|z\|^{\alpha_{k}-1}+\bar{b}_{k}\|u\| \|^{\beta_{k}-1}+\frac{\bar{c}_{k}}{\|z\|+\|u\|}\right\} .
\end{aligned}
$$

Then

$$
\lim _{\||(z, u) \|| \rightarrow \infty} \frac{\left\|\left|\mathcal{S}^{\alpha}(z, u) \|\right|\right.}{\||(z, u) \||}=0
$$


Claim 4. The operator $\mathcal{S}^{\alpha}$ has a fixed point. In fact, for a fixed $0<\rho<1$, there exists $R>0$ big enough such that

$$
\left\|\mathcal{S}^{\alpha}(z, u)\right\||\leqslant \rho\||(z, u)\||,\||(z, u) \||=R .
$$

Hence, if we denote by $B(0, R)$ the ball of center zero and radius $R>0$, we get that $\mathcal{S}^{\alpha}(\partial B(0, R)) \subset B(0, R)$. Since $\mathcal{S}^{\alpha}$ is compact and maps the sphere $\partial B(0, R)$ into the interior of the ball $B(0, R)$, we can apply Rothe's fixed point Theorem 1.2 to ensure the existence of a point $\left(z^{\alpha}, u_{\alpha}\right) \in B(0, R) \subset P C_{t_{1} \cdots t_{P}}\left([0, \tau] ; Z^{1 / 2}\right) \times$ $\mathrm{C}([0, \tau] ; \mathrm{U})$ such that

$$
\left(z^{\alpha}, u_{\alpha}\right)=\mathcal{S}^{\alpha}\left(z^{\alpha}, u_{\alpha}\right)
$$

Claim 5. The sequence $\left\{\left(z^{\alpha}, u_{\alpha}\right)\right\}_{\alpha \in(0,1]}$ is bounded. In fact, for the purpose of contradiction, let us assume that $\left\{\left(z^{\alpha}, u_{\alpha}\right)\right\}_{\alpha \in(0,1]}$ is unbounded. Then, there exits a sub-sequence $\left\{\left(z^{\alpha_{n}}, u_{\alpha_{n}}\right)\right\}_{\alpha \in(0,1]} \subset\left\{\left(z^{\alpha}, u_{\alpha}\right)\right\}_{\alpha \in(0,1]}$ such that

$$
\lim _{n \rightarrow \infty}\left\|\mid\left(z^{\alpha_{n}}, u_{\alpha_{n}}\right)\right\| \|=\infty .
$$

On the other hand, from (4.4) we know for all $\alpha \in(0,1]$ that

$$
\lim _{n \rightarrow \infty} \frac{\left\|\mathcal{S}^{\alpha}\left(z^{\alpha_{n}}, u_{\alpha_{n}}\right)\right\| \mid}{\left\|\left|\left(z^{\alpha_{n}}, u_{\alpha_{n}}\right) \|\right|\right.}=0
$$

Particularly, we have the following situation:

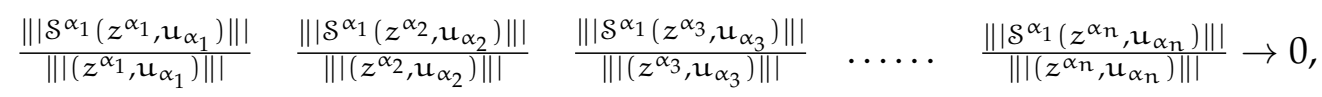

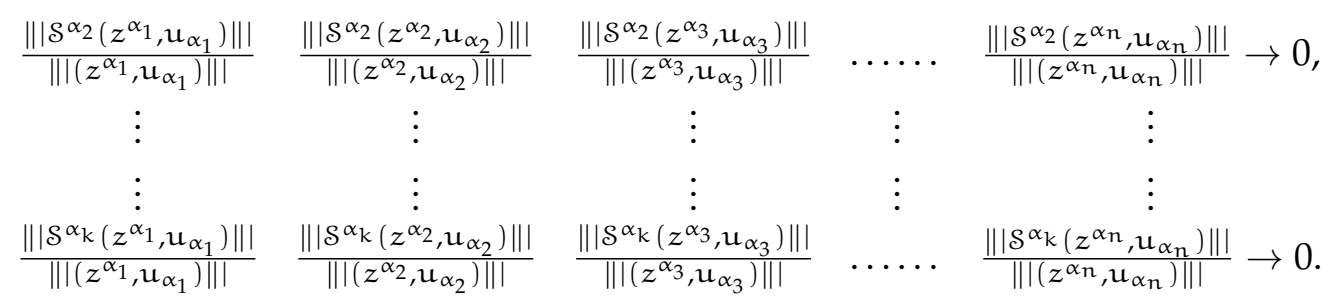

Now, applying Cantor's diagonalization process, we obtain that

$$
\lim _{n \rightarrow \infty} \frac{\left\|\mathcal{S}^{\alpha_{n}}\left(z^{\alpha_{n}}, u_{\alpha_{n}}\right)\right\| \mid}{\|\|\left(z^{\alpha_{n}}, u_{\alpha_{n}}\right) \| \mid}=0
$$

and from (4.5) we have that

$$
\frac{\left\|\mathcal{S}^{\alpha_{n}}\left(z^{\alpha_{n}}, u_{\alpha_{n}}\right)\right\| \mid}{\|\|\left(z^{\alpha_{n}}, u_{\alpha_{n}}\right) \| \mid}=1
$$

which is evidently a contradiction. Then, the claim is true and there exists $l>0$ such that

$$
\left\|\left|\left(z^{\alpha}, u_{\alpha}\right) \|\right| \leqslant l, \quad(0<\alpha \leqslant 1) .\right.
$$

Therefore, without loss of generality, we can assume that the sequence $\mathcal{L}\left(z^{\alpha}, u_{\alpha}\right)$ converges to $y \in Z^{1 / 2}$. So, if

$$
\mathrm{u}_{\alpha}=\Gamma_{\alpha} \mathcal{L}\left(z^{\alpha}, \mathrm{u}_{\alpha}\right)=\mathcal{G}^{*}\left(\alpha \mathrm{I}+\mathcal{G G}^{*}\right)^{-1} \mathcal{L}\left(z^{\alpha}, \mathrm{u}_{\alpha}\right),
$$

Then,

$$
\begin{aligned}
\mathcal{G} \mathrm{u}_{\alpha}=\mathcal{G} \Gamma_{\alpha} \mathcal{L}\left(z^{\alpha}, \mathrm{u}_{\alpha}\right)=\mathcal{G} \mathcal{G}^{*}\left(\alpha \mathrm{I}+\mathcal{G G}^{*}\right)^{-1} \mathcal{L}\left(z^{\alpha}, \mathrm{u}_{\alpha}\right) & =\left(\alpha \mathrm{I}+\mathcal{G} \mathcal{G}^{*}-\alpha \mathrm{I}\right)\left(\alpha \mathrm{I}+\mathcal{G} \mathcal{G}^{*}\right)^{-1} \mathcal{L}\left(z^{\alpha}, \mathrm{u}_{\alpha}\right) \\
& =\mathcal{L}\left(z^{\alpha}, \mathrm{u}_{\alpha}\right)-\alpha\left(\alpha \mathrm{I}+\mathcal{G} \mathcal{G}^{*}\right)^{-1} \mathcal{L}\left(z^{\alpha}, \mathrm{u}_{\alpha}\right)
\end{aligned}
$$

Hence,

$$
\mathcal{G} u_{\alpha}-\mathcal{L}\left(z^{\alpha}, u_{\alpha}\right)=-\alpha\left(\alpha \mathrm{I}+\mathcal{G} \mathcal{G}^{*}\right)^{-1} \mathcal{L}\left(z^{\alpha}, u_{\alpha}\right) .
$$


To conclude the proof of this Theorem, it is enough to prove that

$$
\lim _{\alpha \rightarrow 0}\left\{-\alpha\left(\alpha \mathrm{I}+\mathcal{G G}^{*}\right)^{-1}\right\} \mathcal{L}\left(z^{\alpha}, \mathrm{u}_{\alpha}\right)=0 .
$$

From Lemma $3.2(\mathrm{~d})$, we get that

$$
\begin{aligned}
\lim _{\alpha \rightarrow 0}\left\{\alpha\left(\alpha \mathrm{I}+\mathcal{G} \mathcal{G}^{*}\right)^{-1} \mathcal{L}\left(z^{\alpha}, \mathrm{u}_{\alpha}\right)\right\} & =\lim _{\alpha \rightarrow 0} \alpha\left(\alpha \mathrm{I}+\mathrm{GG}^{*}\right)^{-1} \mathrm{y}+\lim _{\alpha \rightarrow 0} \alpha\left(\alpha \mathrm{I}+\mathcal{G} \mathcal{G}^{*}\right)^{-1}\left(\mathcal{L}\left(z^{\alpha}, \mathrm{u}_{\alpha}\right)-\mathrm{y}\right) \\
& =\lim _{\alpha \rightarrow 0}-\alpha\left(\alpha \mathrm{I}+\mathcal{G} \mathcal{G}^{*}\right)^{-1}\left(\mathcal{L}\left(z^{\alpha}, \mathrm{u}_{\alpha}\right)-\mathrm{y}\right) .
\end{aligned}
$$

On the other hand, from Proposition 3.4, we get that

$$
\left.\left\|\alpha\left(\alpha \mathrm{I}+\mathcal{G G}^{*}\right)^{-1}\left(\mathcal{L}\left(z^{\alpha}, u_{\alpha}\right)-y\right)\right\| \leqslant \| \mathcal{L}\left(z^{\alpha}, u_{\alpha}\right)-y\right) \| .
$$

Therefore, since $\mathcal{L}\left(z^{\alpha}, u_{\alpha}\right)$ converges to $y$, we get that

$$
\lim _{\alpha \rightarrow 0}\left\{-\alpha\left(\alpha \mathrm{I}+\mathcal{G G}^{*}\right)^{-1}\left(\mathcal{L}\left(z^{\alpha}, u_{\alpha}\right)-y\right)\right\}=0 .
$$

Consequently,

$$
\lim _{\alpha \rightarrow 0}\left\{-\alpha\left(\alpha \mathrm{I}+\mathcal{G} \mathcal{G}^{*}\right)^{-1} \mathcal{L}\left(z^{\alpha}, \mathrm{u}_{\alpha}\right)\right\}=0
$$

Then,

$$
\lim _{\alpha \rightarrow 0}\left\{\mathcal{G} \mathbf{u}_{\alpha}-\mathcal{L}\left(z^{\alpha}, \mathbf{u}_{\alpha}\right)\right\}=0
$$

Therefore,

$$
\begin{aligned}
\lim _{\alpha \rightarrow 0}\left\{\mathrm{~T}(\tau) \phi(0)+\mathrm{T}(\tau)\left[\left(g\left(z_{\tau_{1}}, \ldots, z_{\tau_{\mathrm{q}}}\right)\right)(0)\right]+\int_{0}^{\tau} \mathrm{T}(\tau-s) \mathcal{B}_{\omega} \mathrm{u}_{\alpha}(\mathrm{s}) \mathrm{d} s\right. \\
\quad+\int_{0}^{\tau} \mathrm{T}(\tau-\mathrm{s}) \mathcal{F}\left(s, z^{\alpha}(s), z^{\alpha}\left(s-r_{1}\right), \ldots, z^{\alpha}\left(s-r_{\mathrm{m}}\right), \mathrm{u}_{\alpha}(\mathrm{s})\right) \mathrm{d} s \\
\left.\quad+\sum_{0<\mathrm{t}_{\mathrm{k}}<\tau} \mathrm{T}\left(\tau-\mathrm{t}_{\mathrm{k}}\right) \mathcal{J}_{\mathrm{k}}\left(z^{\alpha}\left(\mathrm{t}_{\mathrm{k}}\right), \mathrm{u}_{\alpha}\left(\mathrm{t}_{\mathrm{k}}\right)\right)\right\}=z^{1},
\end{aligned}
$$

and the proof of the theorem is completed.

As a consequence of the foregoing theorem, we can prove the following characterization.

Theorem 4.2. The system (1.1) with impulses, delays, and nonlocal conditions is approximately controllable if for all states $\phi$ and a final state $z^{1}$ and $\alpha \in(0,1]$ the operator $\mathcal{S}^{\alpha}$ given by (4.1)-(4.3) has a fixed point and the sequence $\left\{\mathcal{L}\left(z^{\alpha}, u_{\alpha}\right)\right\}_{\alpha \in(0,1]}$ converges, i.e.,

$$
\left(z^{\alpha}, u_{\alpha}\right)=\mathcal{S}^{\alpha}\left(z^{\alpha}, u_{\alpha}\right), \quad \lim _{\alpha \rightarrow 0} \mathcal{L}\left(z^{\alpha}, u_{\alpha}\right)=y \in Z^{1 / 2} .
$$

\section{Final remark}

Our methodology is simple and can be applied to those second order diffusive processes with impulses, delays, and nonlocal conditions like some control system governed by partial differential equations. For example, the Benjamin-Bona-Mohany Equation with impulses, delays, and nonlocal conditions, the beam equations with impulses, delays, and nonlocal conditions, etc..

Moreover, some of these particular problems can be formulated in a more general setting. Indeed, we can consider the following semilinear evolution equation in a general Hilbert space $Z^{1 / 2}$

$$
\begin{cases}z^{\prime}=\mathcal{A} z+\mathcal{B}_{\omega}+\mathcal{F}\left(\mathrm{t}, z_{\mathrm{t}}, \mathrm{u}(\mathrm{t})\right), & \mathrm{t} \neq \mathrm{t}_{\mathrm{k}}, \\ z(\mathrm{~s})+\left(\mathrm{g}\left(z_{\tau_{1}}, \ldots, z_{\tau_{\mathrm{q}}}\right)\right)(\mathrm{s})=\phi(s), & \mathrm{s} \in[-\mathrm{r}, 0], \\ z\left(\mathrm{t}_{\mathrm{k}}^{+}\right)=z\left(\mathrm{t}_{\mathrm{k}}^{-}\right)+\mathcal{J}_{\mathrm{k}}\left(\mathrm{t}_{\mathrm{k}}, z\left(\mathrm{t}_{\mathrm{k}}\right), \mathrm{u}\left(\mathrm{t}_{\mathrm{k}}\right)\right), & \mathrm{k}=1, \ldots, \mathrm{q}\end{cases}
$$


where $u \in C([0, \tau], U), z=(w, v)^{\top}, \phi=\left(\phi_{1}, \phi_{2}\right)^{\top} \in C([-r, 0], X), z_{t}$ defined as a function from $[-r, 0]$ to $\mathrm{Z}^{1 / 2}$ by $z_{\mathrm{t}}(\mathrm{s})=z(\mathrm{t}+\mathrm{s}),-\mathrm{r} \leqslant \mathrm{s} \leqslant 0$,

$$
\mathcal{A}=\left[\begin{array}{cc}
0 & \mathrm{I}_{\mathrm{X}} \\
-\gamma \mathrm{A} & -\eta A^{1 / 2}
\end{array}\right]
$$

is an unbounded linear operator with domain $\mathrm{D}(\mathcal{A})=\mathrm{D}(A) \times \mathrm{D}\left(\mathrm{A}^{1 / 2}\right)$, IX represents the identity in $X$, and $A: D(A) \subset X \rightarrow Z$ is an unbounded linear operator in $X$ with the following spectral decomposition:

$$
\mathrm{Ax}=\sum_{j=1}^{\infty} \lambda_{j} \sum_{\mathrm{k}=1}^{\gamma_{j}}<z, \phi_{j, k}>\phi_{j, k}
$$

with the eigenvalues $0<\lambda_{1}<\lambda_{2}<\cdots<\cdots \lambda_{n} \rightarrow \infty$ of $A$ having finite multiplicity $\gamma_{j}$ equal to the dimension of corresponding eigenspaces, and $\left\{\phi_{j, k}\right\}$ is a complete orthogonal set of eigenfunctions of $A$. The operator $-A$ generates a strongly continuous compact semigroup $\left\{T_{A}(t)\right\}_{t} \geqslant 0$ given by

$$
\mathrm{T}_{\mathrm{A}}(\mathrm{t}) \mathrm{x}=\sum_{j=1}^{\infty} \mathrm{e}^{-\lambda_{\mathrm{j}} \mathrm{t}} \sum_{\mathrm{k}=1}^{\gamma_{j}}<x, \phi_{j, k}>\phi_{j, k} .
$$

We shall denote by $C$ the space of continuous functions:

$$
C=\left\{\phi:[-r, 0] \rightarrow Z^{1 / 2}: \phi \text { is continuous }\right\},
$$

endowed with the norm

$$
\|\phi\|=\sup _{-r \leqslant s \leqslant 0}\|\phi(s)\|_{Z^{1 / 2}}
$$

The control $u \in C([0, \tau] ; U)$, with $U=Z, B: X \rightarrow X$ is a linear and bounded operator (linear and continuous) and the functions $\mathrm{I}_{\mathrm{k}}^{e}:[0, \tau] \times \mathrm{Z} \times \mathrm{U} \rightarrow \mathrm{Z}, \mathrm{F}:[0, \tau] \times \mathrm{C} \times \mathrm{U} \rightarrow \mathrm{Z}$ satisfy the following inequalities:

$$
\begin{aligned}
\|\mathcal{F}(t, \phi, u)\|_{z} & \leqslant a_{0}\|\phi(-r)\|_{Z}^{\alpha_{0}}+\|u\|_{u}^{\beta_{0}}+c_{0}, \\
\left\|\mathcal{J}_{k}(t, z, u)\right\|_{Z^{1 / 2}} & \leqslant \bar{a}_{k}\|z\|_{Z^{1 / 2}}^{\alpha_{k}}+\bar{b}_{k}\|u\|_{u}^{\beta_{k}}+\bar{c}_{k^{\prime}} \quad k=1, \ldots, p, \\
\left\|g\left(\phi_{1}, \ldots, \phi_{q}\right)\right\|_{C} & \leqslant \sum_{i=1}^{q} \bar{e}_{i}\left\|\phi_{i}\right\|_{C}^{\zeta_{i}}+\bar{e}_{0}, \quad \phi_{i} \in C, \\
\left|g\left(z_{\tau_{1}}, \ldots, z_{\tau_{q}}\right)-g\left(w_{\tau_{1}}, \ldots, w_{\tau_{q}}\right)\right| & \leqslant K\|z-w\|_{P_{t_{1} \ldots t_{p}}\left([-r, \tau] ; Z^{1 / 2}\right)}, \quad z, w \in P_{t_{1} \ldots t_{p}} .
\end{aligned}
$$

In this case the characteristic function set is a particular operator $B$, and the following theorem is a generalization of Theorem 4.1.

Theorem 5.1. If vectors $\mathrm{B}^{*} \phi_{j, k}$ are linearly independent in $\mathrm{Z}^{1 / 2}$, then the system (5.1) is approximately controllable on $[0, \tau]$.

\section{Conclusion}

In this work we prove the interior approximate controllability of the strongly damped equation with impulses, delays, and nonlocal conditions by applying Rothe's fixed point Theorem. After that, we present some open problems and a possible general framework to study the controllability of semilinear second order diffusion process in Hilbert spaces with impulses, delays, and nonlocal conditions. The novelty in this paper is that the literature of control systems with impulses, delays, and nonlocal conditions is very small, there are a very few numbers of papers on systems with impulses, delays, and nonlocal conditions simultaneously. That is to say, control systems governed by partial differential equations with impulses, delays and nonlocal conditions have not been studied much. 


\section{Acknowledgment}

We would like to thank the anonymous referees for their suggestions and comment that help us to improve this paper.

\section{References}

[1] J. Banas, K. Goebel, Measures of Noncompactness in Banach Spaces, Marcel Dekker, New York, (1980). 1.2

[2] A. E. Bashirov, N. I. Mahmudov, On concepts of controllability for deterministic and stochastic systems, SIAM J. Control Optim., 37 (1999), 1808-1821. 3.2

[3] D. N. Chalishajar, Controllability of Impulsive Partial Neutral Funcional Differential Equation with Infinite Delay, Int. J. Math. Anal., 5 (2011), 369-380. 1

[4] L. Z. Chen, G. Li, Approximate Controllability of Impulsive Differential Equations with Nonlocal Conditions, Int. J. Nonlinear Sci., 10 (2010), 438-446. 4

[5] K.-S. Chiu, T. X. Li, Oscillatory and Periodic Solutions of Differential Equations with Piecewise Constant Generalized Mixed Arguments, Math. Nachr., 2019 (2019), 12 pages. 1

[6] R. F. Curtain, A. J. Pritchard, Infinite-dimensional linear systems theory, Springer-Verlag, Berlin, (1978). 3.2

[7] R. F. Curtain, H. Zwart, An Introduction to Infinite Dimensional Linear Systems Theory, Springer-Verlag, New York, (1995). 3.2

[8] C. Guevara, H. Leiva, Controllability of the Impulsive Semilinear Heat Equation with Memory and Delay, J. Dyn. Control Syst., 24 (2018), 1-11. 1

[9] C. Guevara, H. Leiva, Controllability of the Strongly Damped Impulsive Semilinear Wave Equation with Memory and Delay, IFAC J. Syst. Control, 4 (2018), 1-6. 1

[10] G. Isac, On Rothe's Fixed Point Theorem in General Topological Vector Space, An. Ştiinţ. Univ. Ovidius Constanţa Ser. Mat., 12 (2004), 127-134. 1.2

[11] R. S. Jain, M. B. Dhakne, On Mild Solutions of Nonlocal Semilinear Impulsive Functional Integro-Differential Equations, Appl. Math. E-Notes, 13 (2013), 109-119. 4

[12] C. M. Jiang, F. F. Zhang, T. X. Li, Synchronization and Antisynchronization of N-coupled Fractional-Order Complex Chaotic Systems with Ring Connection, Math. Methods Appl. Sci., 41 (2018), 2625-2638. 1

[13] H. Larez, H. Leiva, J. Rebaza, Approximate controllability of a damped wave equation, Can. Appl. Math. Q., 20 (2012), 405-419. 1, 2

[14] H. Leiva, A Lemma on $\mathrm{C}_{0}$-Semigroups and Applications PDEs Systems, Quaest. Math., 26 (2003), 247-265. 2

[15] H. Leiva, Karakostas Fixed Point Theorem and the Existence of Solutions for Impulsive Semilinear Evolution Equations with Delays and Nonlocal Conditions, Commun. Math. Anal., 21 (2018), 68-91. 1, 4, 4

[16] H. Leiva, N. Merentes, J. L. Sánchez, Approximate Controllability of Semilinear Reaction Diffusion, Math. Control Relat. Fields, 2 (2012), 171-182. 3.5

[17] H. Leiva, N. Merentes, J. Sánchez, A Characterization of Semilinear Dense Range Operators and Applications, Abstr. Appl. Anal., 2013 (2013), 11 pages. 3.2, 3.4

[18] H. Leiva, R. Rojas, Controllability of Semilinear Nonautonomous Systems with Impulses and Nonlocal Conditions, Rev. Decienc. Nat., 1 (2016), 23-38. 1

[19] H. Leiva, P. Sundar, Existence of solutions for a class of semilinear evolution equations with impulses and delays, J. Nonlinear Evol. Equ. Appl., 2017 (2017), 95-108. 4

[20] T. X. Li, N. Pintus, G. Viglialoro, Properties of solutions to porous medium problems with different sources and boundary conditions, Z. Angew. Math. Phys., 70 (2019), 18 pages. 1

[21] J. Liang, J. H. Liu, T.-J. Xiao, Nonlocal Impulsive Problems for Nonlinear Differential Equations in Banach Spaces, Math. Comput. Modelling, 49 (2009), 798-804. 1

[22] A. Pazy, Semigroups of Linear Operators and Applications to Partial Differential Equations, Springer-Verlag, New York, (1983). 4

[23] M. H. Protter, Unique continuation for elliptic equations, Trans. Amer. Math. Soc., 95 (1960), 81-91. 3.5

[24] H. Y. Qin, Z. Y. Gu, Y. L. Fu, T. X. Li, Existence of Mild Solutions and Controllability of Fractional Impulsive Integrodifferential Systems with Nonlocal Conditions, J. Funct. Spaces, 2017 (2017), 11 pages. 1

[25] S. Selvi, M. Mallika Arjunan, Controllability Results for Impulsive Differential Systems with Finite Delay, J. Nonlinear Sci. Appl., 5 (2012), 206-219. 1

[26] D. R. Smart, Fixed Point Theorems, Cambridge University Press, London-New York, (1974). 1.2

[27] P. G. Wang, C. R. Li, J. Zhang, T. X. Li, Quasilinearization Method for First-Order Impulsive Integro-Differential Equations, Electron. J. Differential Equations, 2019 (2019), 14 pages. 4 